\title{
Direct signature of light-induced conical
}

\section{intersections in diatomics}

\author{
G. J. Halász, ${ }^{\dagger}$ Á. Vibók, ${ }^{* \neq}$ and L. S. Cederbaum ${ }^{\mathbb{I}}$ \\ Department of Information Technology, University of Debrecen, H-4010 Debrecen, PO Box \\ 12, Hungary, Department of Theoretical Physics, University of Debrecen, H-4010 Debrecen, \\ PO Box 5, Hungary, and Theoretische Chemie, Physikalish-Chemisches Institut, Universität \\ Heidelberg, H-69120, Germany \\ E-mail:vibok@phys.unideb.hu
}

\begin{abstract}
Nonadiabatic effects are ubiquitous in physics, chemistry and biology. They are strongly amplified by conical intersections (CIs) which are degeneracies between electronic states of triatomic or larger molecules. A few years ago it has been revealed that CIs in molecular systems can be formed by laser light even in diatomics. Due to the prevailing strong nonadiabatic couplings, the existence of such laser-induced conical intersections (LICIs) may considerably change the dynamical behavior of molecular systems. By analyzing the photodissociation process of the $\mathrm{D}_{2}^{+}$molecule carefully, we found a robust effect in the angular distribution of the photofragments which serves as a direct signature of the LICI providing undoubted evidence for its existence.
\end{abstract}

\footnotetext{
*To whom correspondence should be addressed

† Department of Information Technology, University of Debrecen, H-4010 Debrecen, PO Box 12, Hungary

$¥$ Department of Theoretical Physics, University of Debrecen, H-4010 Debrecen, PO Box 5, Hungary

ITheoretische Chemie, Physikalish-Chemisches Institut, Universität Heidelberg, H-69120, Germany
} 


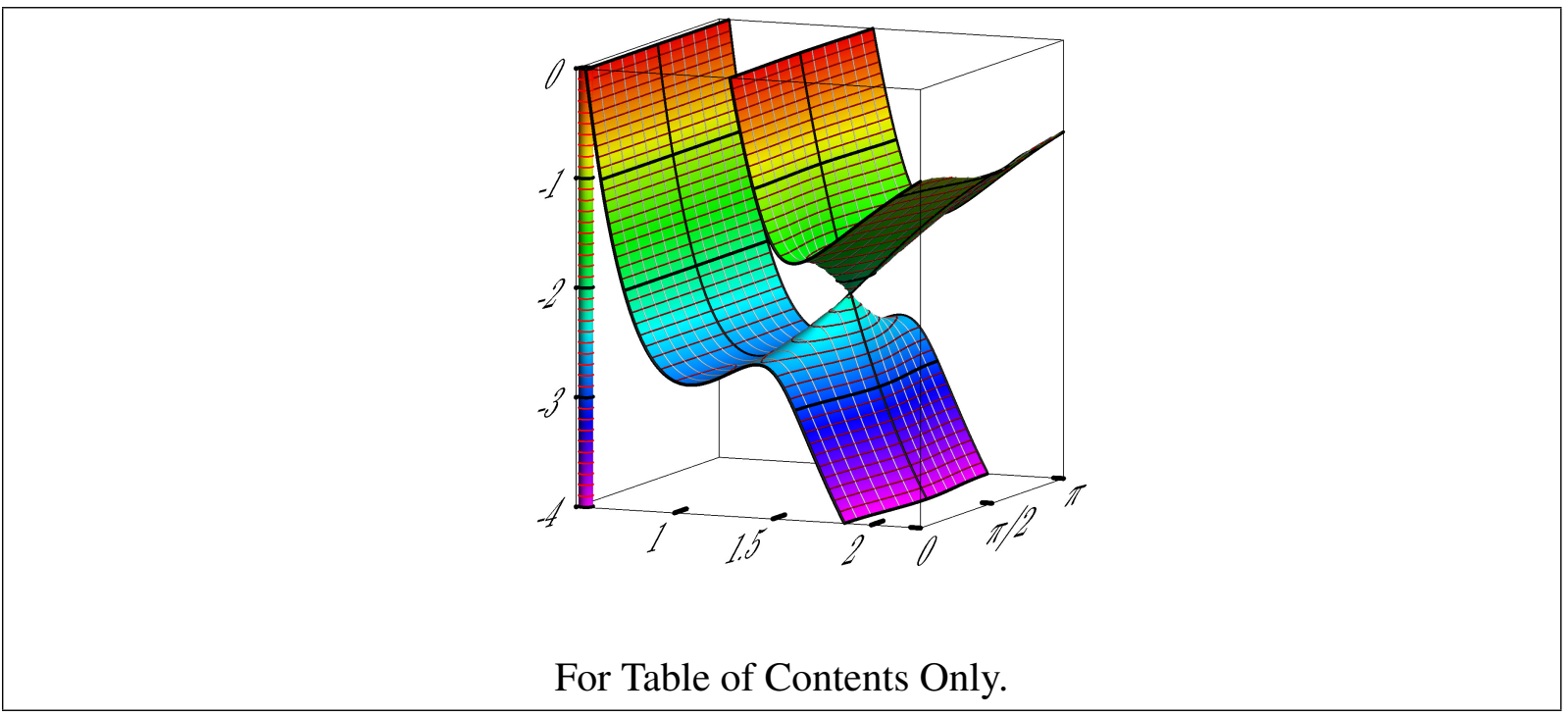

It is well known that conical intersections (CIs) are widely recognized to be ubiquitous in polyatomics, and to play an important role in several different fields like spectroscopy, chemical reaction dynamics, photophysics, photochemistry $1+20$. At CIs the nuclear and electronic motions couple strongly, giving rise to often unexpected - so-called nonadiabatic - phenomena. The nonadiabatic coupling matrix elements between electronic states are the largest possible at CIs where they become singular ${ }^{3}$. Therefore, one expects that such intersections give rise to the strongest possible nonadiabatic phenomena. It was demonstrated for a variety of examples that conical intersections provide the mechanism for ultrafast chemical processes as, e.g. photodissociation, photoisomerization and internal conversion to the electronic ground state ${ }^{9-20}$. Clearly, the CIs must be considered as photochemically relevant decay channels. The presence of CIs in biomolecules, like the building blocks of DNA and proteins contribute to the photostability of these important molecules. CIs can be formed between different electronic states starting from triatomic systems to truly large polyatomic molecules. Several important books, review articles and publications have documented the existence and relevance of such intersections ${ }^{1-20}$.

CIs can exist only if the molecular system possesses at least two independent nuclear degrees of freedom. This is why diatomics which have only one nuclear vibrational coordinate cannot exhibit a CI. Importantly, this statement only holds in field free space, a fact which has been generally overlooked. When a laser field is present, then due to the interaction of the diatomic with this field, the rotational degree of freedom comes into play and serves as an addi- 
tional degree of freedom. It was revealed in earlier studies ${ }^{21 \mid 22}$ that CIs can be induced both by running or standing laser waves even in diatomics. Interestingly, in the standing wave case also the position of the center of mass becomes a new degree of freedom in addition to the nuclear vibrational and rotational motions. In order to demonstrate how the rotation supplies the necessary degree of freedom to facilitate the shaping of a CI in the general case of propagating laser waves, one may adopt the dressed state representation 68169 . In this picture the molecule-light interaction is explicitly included into the Hamiltonian, and the changes of nuclear dynamics due to the light field can be considered as originating from the appearance of a "light-induced conical intersection" (LICI) 22 . The laser frequency determines the position of the LICI, while the laser intensity determines the strength of the nonadiabatic couplings. Our results in the last few years undoubtedly demonstrated that LICIs exert strong effects on the quantum dynamics even for weak laser fields $22-30$.

The present study goes beyond previous investigations and makes an attempt to provide and analyze a physical event which may serve as an undoubted evidence of the laser- or lightinduced conical intersection (LICI), giving a "direct signature" of the presence of this intersection. It is known in the field of nonadiabatic molecular dynamics that due to the extreme breakdown of the Born-Oppenheimer approximation, conical intersections are responsible for ultrafast radiationless processes, typically on the femtosecond time scale. They provide pathways for extremely fast population transfer between electronic states. This latter effect is probably the most important inherent feature of the CIs. Nevertheless, until now one could not find an unambiguous experimentally measurably quantity which reflects directly this population transfer between electronic states for a LICI. The present work discusses a physical process, where an ultrafast population transfer takes place between the electronic states of a diatomic molecule, providing direct evidence for the existence of the LICI. The photodissociation process of the $\mathrm{D}_{2}^{+}$molecule serves as a show case physical example. This molecule has already been studied in vast amount of works $\frac{31+56}{5}$, mainly because of its simplicity. It has the advantage that we can compute it accurately and easily study the light-induced nonadiabatic phenomena separately from other phenomena.

It is important to note that recently, motivated by theoretical predictions on the LICIs in 
diatomics, experiments on the laser-induced isomerization and photodissociation of polyatomic molecules were qualitatively interpreted using the concept of LICIs 5758 . Furthermore, theory devoted to generalize the LICI phenomenon for polyatomics has been derived showing the large potential of LICIs in controlling reactions 59 .

Description of the system. The two relevant electronic states of the $\mathrm{D}_{2}^{+}$ion (see Fig. 1), which will be considered in the calculations are the ground $\left(\mathrm{V}_{1}=1 s \sigma_{g}\right)$ and the first excited $\left(\mathrm{V}_{2}=2 p \sigma_{u}\right)$ eigenstates of the field-free Hamiltonian. For describing the dissociation mechanism we assume that initially the $\mathrm{D}_{2}^{+}$ion is in its ground electronic $\left(1 s \sigma_{g}\right)$ as well as in its ground rotational state and in one of its vibrational eigenstates (see Fig. 1). Exciting the electronic ground state by a resonant laser pulse to the repulsive $2 p \sigma_{u}$ state, the two electronic states are resonantly coupled. The nonvanishing dipole matrix elements are responsible for the light-induced electronic transitions. Within these two electronic states representation the total time-dependent Hamiltonian for the rovibronic nuclear motion reads

$$
\begin{aligned}
& H=\left(\begin{array}{cc}
-\frac{1}{2 \mu} \frac{\partial^{2}}{\partial R^{2}}+\frac{L_{\theta \varphi}^{2}}{2 \mu R^{2}} & 0 \\
0 & -\frac{1}{2 \mu} \frac{\partial^{2}}{\partial R^{2}}+\frac{L_{\theta \varphi}^{2}}{2 \mu R^{2}}
\end{array}\right)+ \\
& \left(\begin{array}{cc}
V_{1}(R) & -\varepsilon_{0} f(t) d(R) \cos \theta \cos \omega_{L} t \\
-\varepsilon_{0} f(t) d(R) \cos \theta \cos \omega_{L} t & V_{2}(R)
\end{array}\right) .
\end{aligned}
$$

Here, $\mathrm{R}$ and $(\theta, \varphi)$ are the molecular vibrational and rotational coordinates, respectively, $\mu$ is the reduced mass, and $L_{\theta \varphi}$ denotes the angular momentum operator of the nuclei. Here $\theta$ is the angle between the polarization direction and the direction of the transition dipole and thus one of the angles of rotation of the molecule. $V_{1}(R)\left(1 s \sigma_{g}\right)$ and $V_{2}(R)\left(2 p \sigma_{u}\right)$ are the potential energies of the two electronic states coupled by the laser (whose frequency is $\omega_{L}$ and amplitude is $\left.\varepsilon_{0}\right), f(t)$ is the envelop function and $d(R)\left(=-\left\langle\psi_{1}^{e}\left|\sum_{j} r_{j}\right| \psi_{2}^{e}\right\rangle\right)$ is the transition dipole matrix element ( $e=m_{e}=\hbar=1$; atomic units are used throughout the article). We used the quantities $V_{1}(R)$ and $V_{2}(R)$ and $d(R)$ published in $\underline{60 \mid 61}$.

Light-induced conical intersection (LICI). In the dressed representation the laser light shifts the energy of the $2 p \sigma_{u}$ repulsive excited potential curve by $\hbar \omega_{L}$ and a crossing between 
the ground $\left(V_{1}\right)$ and the shifted excited $\left(V_{2}-\hbar \omega_{L}\right)$ potential energy curves is created. By diagonalizing the potential energy matrix ${ }^{23}$ one obtains the adiabatic potential surfaces $V_{\text {lower }}$ and $V_{\text {upper }}$ (Fig. 11. These two surfaces cross each other at a single point $R, \theta$, giving rise to a conical intersection whenever the conditions $\cos \theta=0(\theta=\pi / 2)$ and $V_{1}(R)=V_{2}(R)-\hbar \omega_{L}$ are simultaneously fulfilled 22 .

The characteristic properties of the LICI, i.e., the location of the intersections and the strengths of the nonadiabatic couplings, can be directly controlled by the laser frequency and intensity. This opens the possibility to control the nonadiabatic effects emerging from the LICIs. To demonstrate the impact of the LICI on the photodissociation dynamics of $\mathrm{D}_{2}^{+}$we have to solve the time-dependent nuclear Schrödinger equation (TDSE) using the Hamiltonian $\hat{H}$ given by Eq. (??). The angular distribution of the photofragments $P(\theta)$ were calculated with the solution of the TDSE equation.

Methods. The MCTDH (multi configuration time-dependent Hartree) method was used to solve the TDSE 63 . It is one of the most efficient approaches for solving the TDSE. For describing the vibrational degree of freedom we have applied FFT-DVR (Fast Fourier Transformation-Discrete Variable Representation) with $N_{R}$ basis elements distributed within the range from 0.1 a.u. to 80 a.u. for the internuclear separation. The rotational degree of freedom was represented by Legendre polynomials $\left\{P_{J}(\cos \theta)\right\}_{j=0,1,2, \cdots, N_{\theta}}$. These so called primitive basis sets $(\chi)$ were used to build up the single particle functions $(\phi)$, which in turn were applied to represent the wave function:

$$
\begin{aligned}
\phi_{j_{q}}^{(q)}(q, t) & =\sum_{l=1}^{N_{q}} c_{j_{q} l}^{(q)}(t) \chi_{l}^{(q)}(q) \quad q=R, \theta \\
\psi(R, \theta, t) & =\sum_{j_{R}=1}^{n_{R}} \sum_{j_{\theta}=1}^{n_{\theta}} A_{j_{R}, j_{\theta}}(t) \phi_{j_{R}}^{(R)}(R, t) \phi_{j_{\theta}}^{(\theta)}(\theta, t) .
\end{aligned}
$$

In the actual simulations $N_{R}=2048$ and $N_{\theta}=199$ were used. On both diabatic surfaces and for both degrees of freedom a set of $n_{R}=n_{\theta}=20$ single particle functions were applied to construct the nuclear wave packet of the system. The calculations converged properly by using these chosen parameters. Using the solution of equation (2) one can calculate the angular 

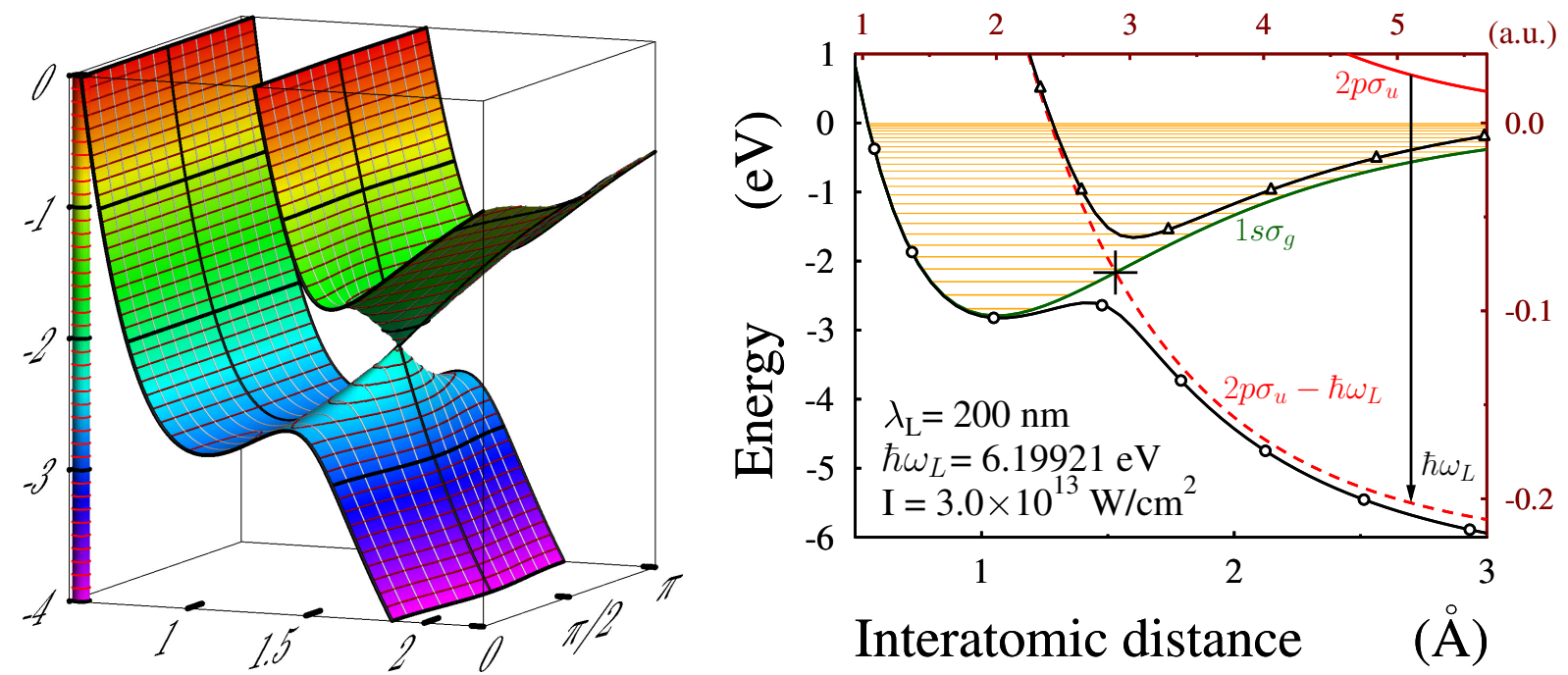

Figure 1: Potential energies of the $\mathrm{D}_{2}^{+}$molecule and the light-induced conical intersection (LICI). Left panel: The dressed adiabatic surfaces as a function of the interatomic distance $R$ and the angle $\theta$ between the molecular axis and the laser polarization exhibiting the LICI for a field intensity of $3 \times 10^{13} \frac{\mathrm{W}}{\mathrm{cm}^{2}}$. Right panel: The diabatic energies of the ground $\left(1 \mathrm{~s} \sigma_{\mathrm{g}}\right)$ and the first excited $\left(2 p \sigma_{u}\right)$ states of the $\mathrm{D}_{2}^{+}$molecule are displayed by solid green and red lines, respectively. The field dressed excited state $\left(2 p \sigma_{u}-\hbar \omega_{L}\right.$; dashed red line) forms a LICI with the ground state. A cut through the adiabatic surfaces at $\theta=0$ (parallel to the field) is depicted by solid black lines marked with circles (lower adiabatic state) and triangles (upper adiabatic state). The position of the LICI is denoted with a cross $\left(R_{\text {LICI }}=1.53 \AA=2.891\right.$ a.u. and $E_{L I C I}=-2.166 \mathrm{eV}$ ). 
distribution of the photofragments $\frac{65}{}$ :

$$
P\left(\theta_{j}\right)=\frac{1}{w_{j}} \int_{0}^{\infty} d t<\psi(t)\left|W_{\theta_{j}}\right| \psi(t)>
$$

where $-i W_{\theta_{j}}$ is the projection of the complex absorbing potential (CAP) on a specific point of the angular grid $\left(j=0, . . N_{\theta}\right)$, and $w_{j}$ is the weight related to this grid point according to the applied DVR.

The simulations. Linearly polarized Gaussian laser pulses centered around $t=0 f s$ were applied in the numerical calculations with a carrier wavelength of $\lambda_{L}=200 \mathrm{~nm}$. The pulse duration at full width of half maximum (FWHM) is $t_{\text {pulse }}=30 \mathrm{fs}$. The initial nuclear wave packet (at $t=-100 f s$ ) was chosen to be in its rotational ground state $(J=0)$ and in one of its vibrational eigenstates $(v=4,5,6,7)$. Under such conditions, the angular distribution of the photofragments can provide accurate details of the photodissociation of single vibrational levels 39 .

Full two dimensional calculations (2d) in $R-\theta$ space have been performed. In order to have a better understanding of the obtained results, we have also performed calculations with a restricted one dimensional (1d) approach where we have eliminated the rotational motion from the system by putting $L_{\theta \varphi}$ to zero in the Hamiltonian Eq. (??). This way the molecule's initial orientation can not change during the dissociation process, and the TDSE can be solved with independent $1 \mathrm{~d}$ calculations for each value of $\theta$ using the "effective field strength" $\varepsilon_{0}^{e f f}=$ $\varepsilon_{0} \cdot \cos \theta$ (intensity $I_{0}^{e f f}=I_{0} \cdot \cos ^{2} \theta$ ) at that value of $\theta$. In these $1 \mathrm{~d}$ calculations the Hamiltonian depends only parametrically on the rotational degree of freedom $(\theta)$ and none of the individual calculations are able to take into account the effects of the LICI. The respective potential energy curves exhibit avoided crossings as can be seen for $\theta=0$ in Fig. 1.

The results obtained for the angular distribution of the dissociation rate for the above described pulse with a peak field intensity of $1 \times 10^{14} \frac{\mathrm{W}}{\mathrm{cm}^{2}}$ are displayed in Figs. $2 \mathrm{a}-2 \mathrm{~d}$ for the four different initial vibrational levels $v=4-7$. The scale on Fig. 2 2 was chosen such that the dissociation rate of 1 implies that the dissociation in a given direction is complete. Consequently, in $1 \mathrm{~d}$ the dissociation rate can not be larger than 1 . Larger values in the full $2 \mathrm{~d}$ calculation mean 

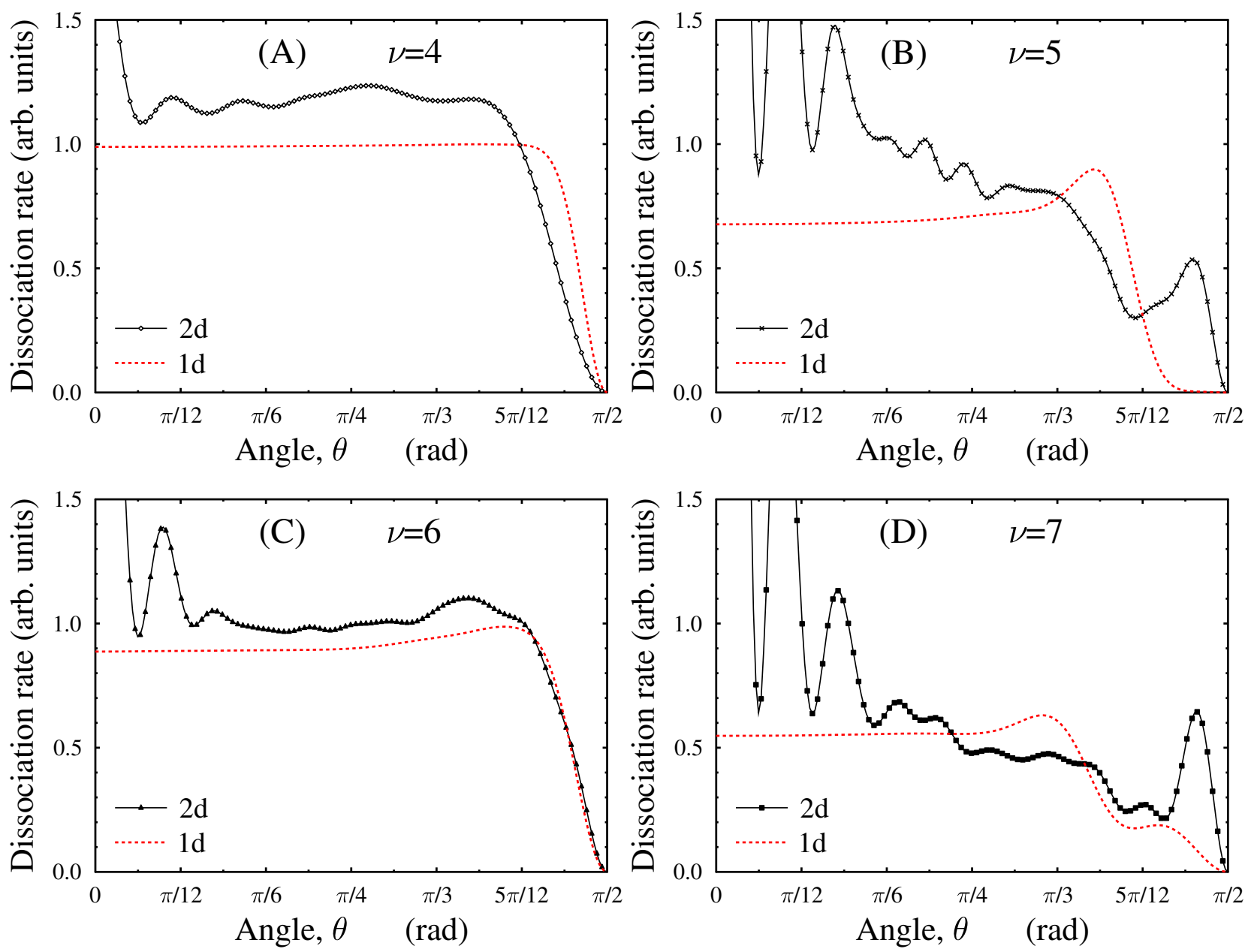

Figure 2: Fragment angular distributions of the dissociating $\mathrm{D}_{2}^{+}$molecule for four different initial vibrational states $(v=4,5,6,7)$. Curves are presented both for one dimensional (1d) and for two dimensional (2d) cases. The applied field intensity is $1 \times 10^{14} \mathrm{~W} / \mathrm{cm}^{2}$. 

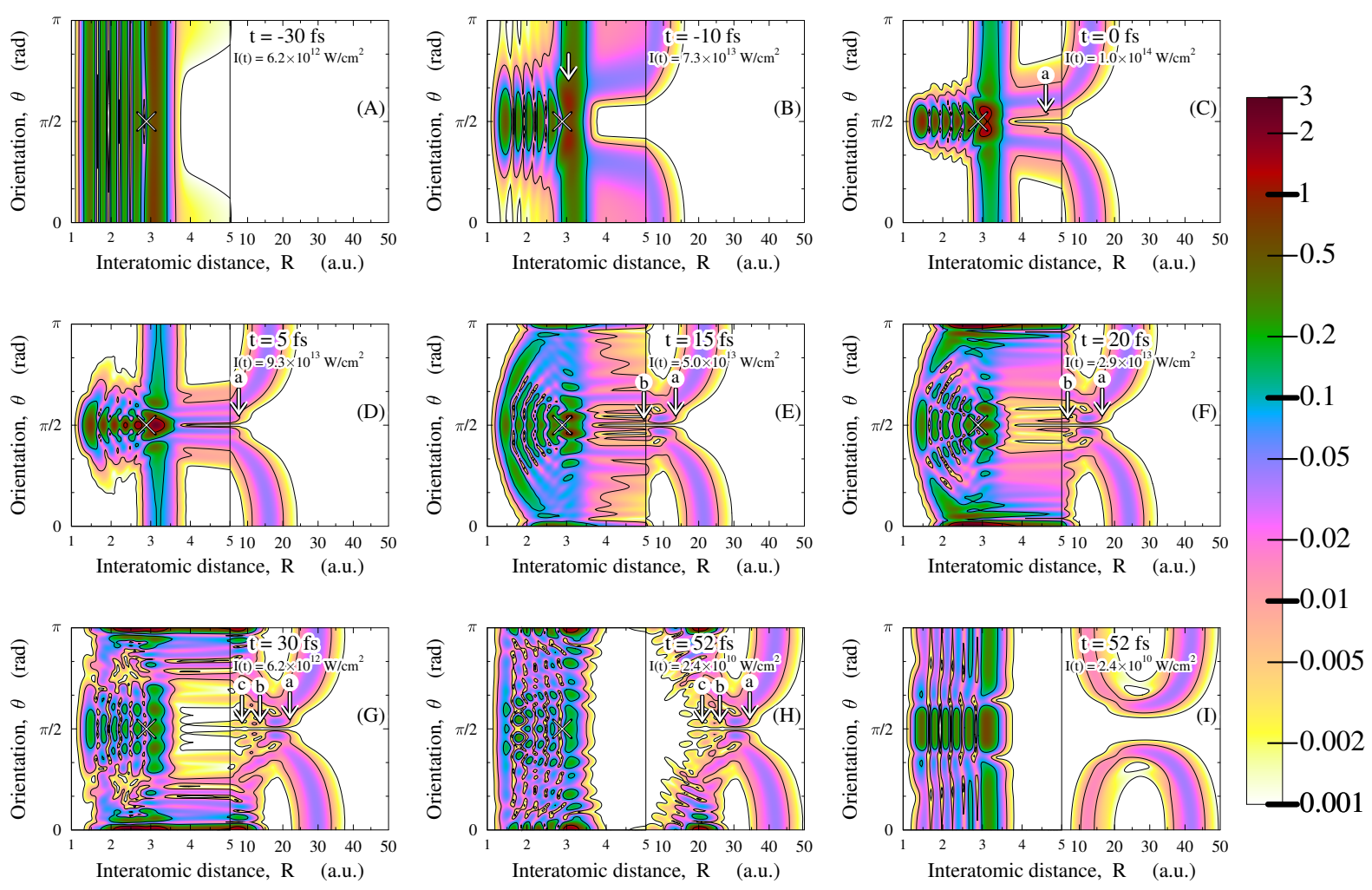

Figure 3: Snapshots from the real-time evolution of the nuclear density of the $v=5$ vibrational state of $\mathrm{D}_{2}^{+}$due to a Gaussian laser pulse of peak intensity $1 \times 10^{14} \mathrm{~W} / \mathrm{cm}^{2}$ and $30 \mathrm{fs}$ duration. The nuclear density exhibits severe interference effects and splits at larger distances around $\theta=\pi / 2$. The instantaneous intensity is shown in the individual snapshots. The yellow cross denotes the position of the LICI. The points $\mathrm{a}, \mathrm{b}$ and $\mathrm{c}$ are explained in the text. All panels show the results of the full $2 \mathrm{~d}$ calculation expect of the last panel (I) which is computed in $1 \mathrm{~d}$ for comparison. Note the jump in the interatomic scale. 
that some parts of the dissociating particles were rotated by the field to this direction from some different initial directions.

Let us study first the $1 \mathrm{~d}$ curves in Fig. 2. For the case of $v=4$ vibrational level the dissociation rate decreases monotonically as a function of $\theta$ and is very close to the value of 1 up to $\theta=5 \pi / 12$. This is related to the fact that the energy of this vibrational level is just above the energy of the LICI and, therefore, most of the initial wavepacket belongs to the lower dissociative adiabatic surface and can easily dissociate. For larger $v$ the $1 \mathrm{~d}$ dissociation rate curves display one or two $(v=7)$ local maxima as a function of the angle $\theta$. This behavior is related to the accidental increase of the so - called bond hardening effect ${ }^{62}$ at particular wavelengths and intensities of the laser field 34 . The $1 \mathrm{~d}$ dissociation rate predicts a significant bond hardening effect for $v=5$ and $v=7$, even at the parallel orientation of the molecules. In a Gaussian laser pulse the field rises smoothly and independently of the peak intensity starts to interact with the molecular system at weak intensities, and therefore the initial populations on the lower and on the upper adiabatic surfaces are determined by the behavior of the system at low intensities. In our earlier work ${ }^{29}$, it was shown for the $v=5$ and $v=7$ initial vibrational levels that the $\lambda_{L}=200 \mathrm{~nm}$ wavelength leads to a close to an "ideal" bond hardening case at weak intensities. This is the reason why we find here an significant bond hardening effect up to $1 \times 10^{14} \frac{\mathrm{W}}{\mathrm{cm}^{2}}$ field intensities at this wavelength.

Let us now turn to the $2 \mathrm{~d}$ results. For the initial $v=4$ vibrational eigenstate, the $2 \mathrm{~d}$ curve (see Fig. 2 2 ) is related to the $1 \mathrm{~d}$ one as one can expect it from the well known light induced potential picture ${ }^{35}$. The differences arise due to our finding that the laser light not just drives the dissociation process, but also starts to rotate the molecules. As a result the dissociation rate decreases at large $\theta$ angles and at the same time increases at the small values of $\theta$. As the induced rotation towards the polarization direction of the electric field creates rotational nodes, the angular distribution of the dissociation rate is further affected by the appearance of additional structures 28 .

For the $v=6$ vibrational level (see Fig. 22) the increase of the dissociation rate at small angles is not accompanied by its decrease at orientations close to the direction perpendicular to the polarization axis. Actually, very close to $\theta=\pi / 2$ the dissociation rate in $2 \mathrm{~d}$ is slightly 
larger than in $1 \mathrm{~d}$. This may be interpreted as follows. The population of the upper adiabatic surface is rotated by the electric field into this direction and later on this extra population can dissociate by population transfer to the lower adiabatic surface in the vicinity of the LICI.

From this explanation we may expect more pronounced effects for the $v=5$ and $v=7$ cases, where the bond hardening is more effective as concluded from the study in $1 \mathrm{~d}$. Indeed, in these cases the $2 d$ dissociation rates in Fig. 2 exhibit large peaks around $\theta \gtrsim 11 \pi / 24$, whose heights are one $(v=7)$ or even two $(v=5)$ orders of magnitude larger than the corresponding dissociation rates in the $1 \mathrm{~d}$ calculations. This characteristic structure of the $2 \mathrm{~d}$ curves in this angle region can not be explained without the strong nonadiabatic effect due to the existence of the LICI. The peaks displayed on the $2 \mathrm{~d}$ curves are direct fingerprints of the existence of the laser-induced conical intersection in the studied system. We note here that such peaks can also be seen at smaller intensities $\left(1 \times 10^{13} \frac{\mathrm{W}}{\mathrm{cm}^{2}}, 3 \times 10^{13} \frac{\mathrm{W}}{\mathrm{cm}^{2}}\right)$, but in less pronounced form.

To understand the underlying dynamical process more deeply, we analyzed the nuclear density function $|\psi(R, \theta, t)|^{2}\left(=\left|\psi^{1 s \sigma_{g}}(R, \theta, t)\right|^{2}+\left|\psi^{2 p \sigma_{u}}(R, \theta, t)\right|^{2}\right)$. Snapshots of the nuclear wave packet density functions are shown in Fig. 3. After the pulse is over $(t=52 f s$, Fig. 3h) we can identify three peaks - on both sides of the symmetry axis $\theta=\pi / 2$ - in the region of interest $11 \pi / 24 \lesssim \theta \lesssim 13 \pi / 24$ of the outgoing wavepacket. These peaks - especially the most right one - are clearly responsible for the characteristic structure of the huge excess in the dissociation probability close to $\theta=\pi / 2$. Their "leading edge" are labeled by "a", "b" and "c" on the subfigures whenever they are recognizable.

In what follows we study the time evaluation of the nuclear density in order to see the origin of these peaks. It can be seen that at $t=-30 f s$ (Fig. 3a) the molecules start to dissociate on the lower adiabatic surface. This process evolves in time, but at $t=-10 f s$ one can clearly recognize also the fingerprint of the rotational motion induced on the upper surface (see the arrow in Fig. 3b). At $t=0 f s$ (Fig. 3r, labeled by "a") tracks show up that an additional dissociation process starts close to $\theta=\pi / 2$, presumably from the upper adiabatic surface. From $t=15 \mathrm{fs}$ (Fig. 3e) on it is clearly seen that additional dissociation takes place continuously, and from $t=20 f s$ (Fig. 3f) on the dissociated fragments explicitly appear in the dissociation region $(R>5 a . u$.$) . The much less populated "b" and "c" peaks appear for the first time on subfigures$ 
Fig. 3, and Fig. 3g, respectively.

The last snapshot displays the results of the $1 \mathrm{~d}$ simulation at $t=52 \mathrm{fs}$, the same time as in Fig. 3h for the full $2 \mathrm{~d}$ calculation. The dynamics during the initial time period, when the instantaneous intensity is low, are very similar in both the $1 \mathrm{~d}$ and $2 \mathrm{~d}$ models. After reaching the maximum intensity, however, two significant differences can be observed. The first one is, as expected, the unequivocal fingerprint of the presently discussed nonadiabatic effect leading to a strong enhancement of fragment density near $\theta=\pi / 2$ in the $2 \mathrm{~d}$ model, while the second one is the appearance of interference patterns in the $2 \mathrm{~d}$ model. As both effects rely on the dissociation of those molecules which have been previously rotated by the field, the $1 \mathrm{~d}$ model is unable to account for them. On the other hand, it is not surprising that at the beginning of the dissociation process, when the rotation develops slowly, the $1 \mathrm{~d}$ and $2 \mathrm{~d}$ approaches lead to the similar smooth behavior in the dissociation region.

In summary, the results obtained clearly show the direct impact of the laser-induced conical intersection on the dissociation dynamics of the $\mathrm{D}_{2}^{+}$molecule. The structure and magnitude of the $2 \mathrm{~d}(v=5,7)$ dissociation rates close to $\theta=\pi / 2$ undoubtedly demonstrate the strong nonadiabatic effects due to the presence of the LICI. The strong nonadiabaticity turns the molecules perpendicular to the polarization direction and then these molecules can travel through the LICI to the lower adiabatic surface on which they dissociate. This process can only happen if population transfer takes place via the laser-induced conical intersection.

\section{Acknowledgements}

The authors acknowledge financial support by the Deutsche Forschungsgemeinschaft (Project ID CE10/50-2). Á. V. also acknowledges the TÁMOP-4.2.4.A/ 2-11/1-2012-0001 'National Excellence Program' and the OTKA (NN103251) project. This research was supported in part by the National Science Foundation under Grant No. NSF PHY11-25915. 


\section{References}

(1) Zener, C. Non-adiabatic crossing of energy level. Proc. R. Soc. Lond. A 1932, 137,696702.

(2) Teller, E. The crossing of potential energy surfaces. J. Phys. Chem. 1937, 41, 109-116.

(3) Köppel, H.; Domcke, W.; Cederbaum L. S. Multimode molecular dynamics beyond the Born-Oppenheimer approximation. Adv. Chem. Phys. 1984, 57, 59-246.

(4) Truhlar, D. G.; Mead, A. The relative likelihood of encountering conical intersections on the potential energy surfaces of polyatomic molecules. Phys. Rev. A 2003, 68, 032501.

(5) Baer, M. Introduction to the theory of electronic non-adiabatic coupling terms in molecular system. Phys. Rep. 2002, 358, 75-142.

(6) Worth, G. A.; Cederbaum, L. S. Beyond Born-Oppenheimer: Molecular dynamics through a conical intersection. Annu. Rev. Phys. Chem. 2004, 55, 127-158.

(7) Domcke, W.; Yarkony, D. R.; Köppel, H. Conical Intersections: Electronic Structure, Dynamics and Spectroscopy 2004, (Singapore: World Scientific).

(8) Baer, M. Beyond Born Oppenheimer: Electronic Non-Adiabatic Coupling Terms and Conical Intersections 2006, (New York: Wiley).

(9) Matsika, S. Conical intersections in molecular systems. Rev. Comput. Chem. 2007, 23, 83-124.

(10) Matsika, S.; Krause, P. Nonadiabatic events and conical intersections. Annu. Rev. Phys. Chem. 2011, 62, 621-43.

(11) Kling, M. F.; Siedschlag, Ch.; Verhoef, A. J.; Khan, J. I.; Schultze, M.; Uphues, Th.; Uiberacker, Y. Ni. M.; Drescher, M.; Krausz, F.; Vrakking, M. J. J. Control of Electron Localization in Molecular Dissociation, Science 2006, 312, 246-248.

(12) Ashfold, M. N. R. et al. The role of $\pi \sigma^{*}$ excited state in the photodissociation of heteroaromatic molecules. Science 2006, 312, 1637-1640. 
(13) Ashfold, M. N. R. et al. Exploring nuclear motion through conical intersections in the UV photodissociation of phenols and thiophenol. PNAS 2008, 105, 12701-12706.

(14) Lim, J. S.; Kim, S. K. Experimental probing of conical intersection dynamics in the photodissociation of thioanisole. Nature Chemistry 2010, 2, 627-632.

(15) Polli, D. et al. Conical intersection dynamics of the primary photoisomerization event in vision. Nature 2010, 467, 440-443.

(16) Gozem, S.; Krylov, A.I.; Olivucci, M. Conical intersection and potential energy surface features of a model retinal chromophore: Comparison of EOM-CC and multireference methods. J. Chem. Theory Comput. 2013, 9, 284-292.

(17) Martinez, T. J. Seaming is believing. Nature 2010, 467, 412-413.

(18) Wörner, H. J. et al. Conical Intersection Dynamics in $\mathrm{NO}_{2}$ Probed by Homodyne HighHarmonic Spectroscopy, Science 2011, 334, 208-2012.

(19) Zhou, X. et al. Probing and controlling non-Born-Oppenheimer dynamics in highly excited molecular ions Nature Physics 2012, 8, 232-237.

(20) Gozem, S. et al. Shape of multireference, equation-of-motion coupled-cluster, and density functional theory potential energy surfaces at a conical intersection. J. Chem. Theory Comput. 2014, 10, 3074-3084.

(21) Moiseyev, N.; Sindelka, M.; Cederbaum, L. S. Laser-induced conical intersection in molecular optical lattices. J. Phys B. 2008, 41, 221001-5.

(22) Sindelka, M.; Moiseyev, N.; Cederbaum, L. S. Strong impact of light-induced conical intersections on the spectrum of diatomic molecules. J. Phys B. 2011, 44, 045603-6.

(23) Halász, G. J.; Vibók, Á.; Sindelka, M.; Moiseyev, N.; Cederbaum, L. S. Conical Intersections Induced by Light: Berry phase and Wavepacket Dynamics. J. Phys B. 2011, 44, 175102-12. 
(24) Halász, G. J.; Sindelka, M.; Moiseyev, N.; Cederbaum, L. S.; Vibók, Á. Light-induced conical intersections: Topological phase, wave packet dynamics, and molecular alignment. J. Phys. Chem. A 2012, 116, 2636-2643.

(25) Halász, G. J.; Vibók, Á.; Sindelka, M.; Cederbaum, L. S.; Moiseyev, N. The effect of light-induced conical intersections on the alignment of diatomic molecules. Chem. Phys. 2012, 399, 146-150.

(26) Halász, G. J.; Vibók, Á.; Moiseyev, N.; Cederbaum, L. S. Light-induced conical intersections for short and long laser pulses: Floquet and rotating wave approximations versus numerical exact results. J. Phys. B 2012, 45, 135101-10.

(27) Halász, G. J.; Vibók, Á.; Meyer, H. D.; Cederbaum, L. S. Effect of light-induced conical intersection on the photodissociation dynamics of the $\mathrm{D}_{2}^{+}$molecule. J. Phys. Chem. A 2013, 117, 8528-8535.

(28) Halász, G. J.; Vibók, Á.; Moiseyev, N.; Cederbaum, L. S. Nuclear-wave-packet quantum interference in the intense laser dissociation of the $\mathrm{D}_{2}^{+}$molecule. Phys. Rev. A 2013, 88, 043413-6.

(29) Halász, G. J.; Csehi, A.; Vibók, Á.; Cederbaum, L. S.; The influence of light-induced conical intersection on the photodissociation dynamics of $\mathrm{D}_{2}^{+}$from single vibrational levels. J. Phys. Chem. A. 2014, dx.doi.org/10.1021/jp504889e:1-8.

(30) Cederbaum, L. S.; Chiang, Y. C.; Demekhin, P. V.; Moiseyev, N. Resonant auger decay of molecules in intense X-Ray fields: Light-induced strong nonadiabatic effects. Phys. Rev. Lett. 2011, 106, 123001-4.

(31) Zavriyev, A.; Bucksbaum, P. H.; Muller, H. G.; Schumacher, D. V. Ionization and dissociation of $\mathrm{H}_{2}$ in intense laser fields at $1.064 \mu \mathrm{m}, 532 \mathrm{~nm}$, and $355 \mathrm{~nm}$. Phys. Rev. A 1990, 42, 5500-3513.

(32) Bandrauk, A. D.; Sink, M. Laser induced preassociation in the presence of natural predissociation. Chem. Phys. Lett. 1978, 57, 569-572. 
(33) Bandrauk, A. D.; Sink, M. Photodissociation in intense laser fields: Predissociation analogy. J. Chem. Phys. 1981, 74, 1110-1117.

(34) Aubanel, E. E.; Gauthier, J. M.; Bandrauk, A. D. Effect of rotations on stabilization in high-intensity photodissociation of $\mathrm{H}_{2}^{+}$. Phys. Rev. A 1993, 48, 2145-2152.

(35) Charron, E.; Giusti-Suzor, A.; Mies, F. H. Fragment angular distribution in one- and twocolor photodissociation by strong laser fields. Phys. Rev. A 1994, 49, R641-R644.

(36) Chelkowski, S.; Zuo, T.; Atabek, O.; Bandrauk, A. D. Dissociation, ionization, and coulomb explosion of $\mathrm{H}_{2}^{+}$in an intense laser field by numerical integration of the timedependent Schrödinger equation. Phys. Rev. A 1995, 52, 2977-2983.

(37) Giusti-Suzor, A.; Mies, F. H.; DiMauro, L. F.; Charron, E.; Yang, B. Dynamics of $\mathrm{H}_{2}^{+}$in intense laser fields. J. Phys B. 1995, 28, 309-339.

(38) Numico, R.; Keller, A.; Atabek, O. Laser-induced molecular alignment in dissociation dynamics. Phys. Rev. A 1995, 52, 1298-1309.

(39) Sandig, K.; Figger, H.; Hansch, T. V. Dissociation dynamics of $\mathrm{H}_{2}^{+}$in intense laser fields: Investigation of photofragments from single vibrational levels. Phys. Rev. Lett. 2000, 85, 4876-4879.

(40) Serov, V. N.; Keller, A.; Atabek, O.; Billy, N. Quantitative theory-versus-experiment comparison of the intense laser dissociation of $\mathrm{H}_{2}^{+}$. Phys. Rev. A 2003, 68, 053401-16.

(41) Posthumus, J. H. The dynamics of small molecules in intense laser fields. Rep. Prog. Phys. 2004, 67, 623-665.

(42) Serov, V. N.; Keller, A.; Atabek, O.; Figger, H.; Pavidic, D. Intense laser dissociation of $\mathrm{D}_{2}^{+}$: From experiment to theory. Phys. Rev. A 2005, 72, 033413-21.

(43) Uhlmann, M.; Kunert, T.; Schmidt, R. Molecular alignment of fragmenting $\mathrm{H}_{2}^{+}$and $\mathrm{H}_{2}$ in strong laser fields. Phys. Rev. A 2005, 72, 045402-5. 
(44) Wang, P. Q. et al. Dissociation of $\mathrm{H}_{2}^{+}$in intense femtosecond laser fields studied by coincidence three-dimensional momentum imaging. Phys. Rev. A 2006, 74, 043411-21.

(45) Anis, F.; Esry, B. D. Role of nuclear rotation in dissociation of $\mathrm{H}_{2}^{+}$in a short laser pulse. Phys. Rev. A 2008, 77, 033416-11.

(46) Anis, F.; Cackowski, T.; Esry, B. D. Rotational dynamics of dissociating $\mathrm{H}_{2}^{+}$in a short intense laser pulse. J. Phys B. Fast. Track Com. 2009, 42, 091001-6.

(47) Hua, J. J.; Esry, B. D. Laser-induced multiphoton dissociation branching ratios for $\mathrm{H}_{2}^{+}$ and $\mathrm{D}_{2}^{+}$. Phys. Rev. A. 2009, 80, 013413-1-9.

(48) Adhikari, S. et al. Molecular systems in intense short-pulsed external fields: Application of space-time contours for $\mathrm{H}_{2}^{+}$. J. Phys. Chem. A 2009, 113, 7331-7337.

(49) Paul, A. K.; Adhikari, S.; Baer, M.; Baer, R. $\mathrm{H}_{2}^{+}$Photodissociation by an intense pulsed photonic Fock state. Phys. Rev. A 2010, 81, 013412-10.

(50) Thumm, U.; Niederhausen, T.; Feuerstein, B. Time-series analysis of vibrational nuclear wave-packet dynamics in $\mathrm{D}_{2}^{+}$. Phys. Rev. A 2008, 77, 063401-12.

(51) Fischer, M. et al. Mixed quantum-classical approach to multiphoton dissociation of the hydrogen molecular ion. New. J. Phys. 2011, 13, 053019-14.

(52) Fischer, M.; Lorenz, U.; Schmidt, B.; Schmidt, R. Fragmentation due to centrifugal forces in the photodissociation of $\mathrm{H}_{2}^{+}$in intense laser fields. Phys. Rev. A 2011, 84, 033422-5.

(53) McKenna, J. et al. Controlling strong-field fragmentation of $\mathrm{H}_{2}^{+}$by temporal effects with few-cycle laser pulses. Phys. Rev. A 2012, 85, 023405-15.

(54) He, H. X.; Lu, R. F.; Zhang, P. Y.; Han, K. L.; He, G. Z. Dissociation and ionization competing processes for $\mathrm{H}_{2}^{+}$in intense laser field: Which one is larger? J. Chem. Phys. 2012, 136, 024311-6.

(55) Furukawa, Y.; et al. Resolving vibrational wave-packet dynamics of $\mathrm{H}_{2}^{+}$using multicolor probe pulses. Opt. Lett. 2012, 37, 2922-2924. 
(56) Handt, J. et al. Complete dynamics of $\mathrm{H}_{2}^{+}$in strong laser fields. 2011, $\operatorname{arXiv:1103.1565\mathrm {v}2.}$

(57) Kim, J. et al. Control of 1,3-Cyclohexadiene Photoisomerization Using Light-Induced Conical Intersections. J. Phys. Chem. A 2012, 116, 2758-2763.

(58) Corrales, M. E. et al. Control of Ultrafast Molecular Photodissociation by Laser-FieldInduced Potentials. Nature Chem. 2014, doi: 10.1038/nchem.2006, 1-6.

(59) Demekhin, P. V.; Cederbaum, L. S. Light-induced conical intersections in polyatomic molecules: General theory, strategies of exploitation, and application. J. Chem. Phys. 2013, 139, 154314-15.

(60) Bunkin, F. V.; Tugov, I. I. Multiphoton processes in homopolar diatomic molecules. Phys. Rev. A 1973, 8, 601-612.

(61) Chu, S. I.; Laughlin, C.; Datta, K. Two-photon dissociation of vibrationally excited $\mathrm{H}_{2}^{+}$. Complex quasi-vibrational energy and inhomogeneous differential equation approaches. Chem. Phys. Lett. 1983, 98, 476-481.

(62) Bandrauk, A. D.; Aubanel, E.; Gauthier, J. M. Molecules in Laser Fields (Marcel Dekker Inc., New York) 1994.

(63) Meyer, H. D.; Manthe, U.; Cederbaum, L. S. The Multi-Configurational Time-Dependent Hartree Approach. Chem. Phys. Lett. 1999, 165, 73-78.

(64) Manthe, U.; Meyer, H. D.; Cederbaum, L. S. Wave-packet dynamics within the multiconfiguration Hartree framework: General aspects and application to NOCl. J. Chem. Phys. 1992, 97, 3199-3213.

(65) Beck, M. H.; Jäckle, A.; Worth, G. A.; Meyer, H. D. The Multiconfiguration timedependent Hartree method: A highly efficient algorithm for propagating wavepackets. Phys. Rep. 2000, 324, 1-105.

(66) Worth, G. A. et. al. The MCTDH package, version 8.4, University of Heidelberg, Germany; http://mctdh.uni-hd.de/ 2007. 
(67) Meyer, H. D.; Gatti, F.; Worth, G. A. Multidimensional Quantum Dynamics: MCTDH Theory and Applications (Weinheim: Wiley-VCH) 2009.

(68) Shirley, J. H. Solution of the Schrodinger equation with a Hamiltonian periodic in time. Phys. Rev. 1965, 138, B979-B987.

(69) Chu, S. I. Floquet theory and complex quasivibrational energy formalism for intense field molecular photodissociation J. Chem. Phys. 1981, 75, 2215-2221. 